\title{
ARTÍCULOS
}

\section{EL USO DEL PODCAST PARA LA DIFUSIÓN DEL PATRIMONIO CULTURAL EN EL ENTORNO HISPANOPARLANTE: ANÁLISIS DE LAS PLATAFORMAS IVOOX Y SOUNDCLOUD.}

\author{
David Parra Valcarce \\ Universidad Complutense de Madrid \\ davidparra@ccinf.ucm.es \\ Charo Onieva Mallero \\ Viva Voz \\ onieva@cincuentopia.com
}

Resumen: La tecnología del podcasting ha conocido un considerable crecimiento durante estos últimos años, facilitada por circunstancias como la consolidación de la web semántica manual o web 2.0 y el surgimiento de la figura del prosumidor de contenidos. Desde el punto de vista de su evolución en el tiempo consideramos la existencia de cuatro etapas diferenciadas, a las que denominamos como experimental (finales del siglo XX a 2005), de consolidación (2006 a 2009), multimedia (2010 a 2013) y de eclosión (2014 hasta la actualidad). Se analiza cuál es el grado de utilización de los podcasts para el tratamiento de la temática referida a la difusión del patrimonio cultural en el ámbito específico hispanoparlante. Para ello, se ha examinado la presencia de esta clase de herramientas en dos de las principales plataformas de podcasting a escala internacional: iVoox y SoundCloud.

Palabras clave: Podcast, podcasting, patrimonio cultural, iVoox, SoundCloud, web 2.0.

Tittle: PODCAST USE FOR SPREADING CULTURAL HERITAGE IN HISPANIC-SPEAKING ENVIRONMENT: ANALYSIS OF IVOOX AND SOUNDCLOUD PLATFORMS.

Abstract: Podcasting technology has had a considerable growth during last years, facilitated by circumstances such as the consolidation of the manual semantic web or web 2.0 and the emergence of the figure of the prosumer of contents. From the point of view of its chronological evolution, we consider the existence of four differentiated stages, which we call as experimental (late 20th century to 2005), consolidation (2006 to 2009), multimedia (2010 to 2013) and hatching (2014 to nowadays). We analyze the degree of use of podcasts for the treatment of the theme related to the dissemination of cultural heritage in the specific Hispanic-speaking environment. In order to do so, we have examined the presence of this kind of tools in two of the main international podcasting platforms: iVoox and SoundCloud.

Keywords: Podcast, podcasting, cultural heritage, iVoox, SoundCloud, web 2.0.

Recibido: $17-02-2020$

Aceptado: $19-02-2020$

Cómo citar este artículo: PARRA VALCARCE, David y ONIEVA MALLERO, Charo. El uso del podcast para la difusión del patrimonio cultural en el entorno hispanoparlante: análisis de las plataformas iVoox y SoundCloud. Naveg@mérica. Revista electrónica editada por la Asociación Española de Americanistas [en línea]. 2020, n. 24. Disponible en: <http://revistas.um.es/navegamerica>. [Consulta: Fecha de consulta]. ISSN 1989-211X. 


\section{Introducción y objetivos}

El término podcast es acuñado por vez primera por Hammersley en 2004 a partir de la combinación de las palabras iPod y broadcasting, identificándolo en un primer momento con una herramienta con capacidad para producir contenidos de índole radiofónica y proceder a su distribución mediante suscripción a través de internet. En ese artículo dicho autor muestra sus dudas a la hora de establecer tal denominación y esboza la posibilidad de emplear otras opciones como audioblogging o incluso GuerillaMedia ${ }^{1}$.

No obstante experiencias de esta índole ya se llevaban a cabo desde finales de la última década del siglo $\mathrm{XX}$, principalmente mediante el empleo de funcionalidades RSS de sindicación de contenidos ${ }^{2}$, por parte de algunas emisoras de Estados Unidos especializadas en música pop con el objetivo fundamental de ampliar la cobertura de su programación a determinados segmentos poblacionales que encontraban dificultades para acceder a los ondas hertzianas emitidas a través del espectro radioeléctrico.

Es precisamente la tecnología RSS la que permitirá meses después de la formulación del concepto que Adam Curry, pinchadiscos que trabajaba en la cadena estadounidense MTV, incorporara archivos de audio que eran así distribuidos entre la comunidad internauta de manera más eficaz, generando una verdadera agrupación de usuarios que no se limitaba a emitir opiniones sobre dichos contenidos sino que podía compartir y recomendar los mismos, según subraya Sellas Güell ${ }^{3}$.

Este artículo forma parte de una investigación que plantea como objetivo principal el análisis de cuál es el peso específico de los podcasts, centrados temática y expresamente en la difusión del patrimonio cultural, dentro del entorno castellano parlante compuesto en la actualidad por un total de $\mathbf{5 8 0}$ millones de personas, de las que 483 millones tienen el español como lengua nativa, tal y como se pone de manifiesto en el último de los informes elaborados por el Instituto Cervantes ${ }^{4}$.

A fecha de hoy, según explicita ese mismo informe, el idioma castellano es el segundo más hablado en el mundo, el tercero más empleado en internet y cuenta con casi 22 millones de personas que lo estudian en 110 países. Las previsiones

\footnotetext{
${ }^{1}$ HAMMERSLEY, Ben. Audible revolution. The Guardian [en línea]. 2004, 12 de febrero. [Fecha de consulta: 05-01-2020]. Disponible <https://www.theguardian.com/media/2004/feb/12/broadcasting.digitalmedia>.

2 RSS son las siglas que corresponden a Really Simple Syndication. Su surgimiento se enmarca en una innovación tecnológica inicialmente desarrollada por la compañía Netscape en 1999 con la que trataba de que los internautas usuarios de su motor de navegación estuvieran en condiciones de acceder a contenidos de muy variada procedencia desde un sitio único.

${ }^{3}$ SELLAS GÜELL, Antoni. La voz de la Web 2.0. Análisis del contexto, retos y oportunidades del podcasting en el marco de la comunicación sonora [en línea]. Barcelona: Universitat Internacional de Catalunya, 2009. 418 p. ISBN: 9788469197929. [Fecha de consulta: 19-02-2020]. Disponible en $<$ https://www.tdx.cat/handle/10803/9351\#page=1>.

4 Instituto Cervantes. El español: una lengua viva. Informe 2019 [en línea]. Madrid: Instituto Cervantes, 2019. 96 p. [Fecha de consulta: 19-02-2020]. Disponible en <https://www.cervantes.es/imagenes/File/espanol_lengua_viva_2019.pdf>.
} 
estiman que el peso de la comunidad hispano hablante en 2050 alcanzará el 7,7 por ciento de la población mundial y que en 2060 Estados Unidos se convertirá en el segundo país castellano parlante del mundo, después de México, con casi uno de cada tres estadounidenses.

Se considera que en un entorno de tales características el conocimiento del patrimonio cultural de esta gran comunidad deberá ser consolidado mediante su activa difusión a partir del empleo de distintas herramientas estratégicas, incluyendo las nuevas tecnologías de la información y las comunicaciones en general y el podcast en particular, por lo que la reflexión sobre su estado actual y posibilidades de evolución en el tiempo resulta particularmente pertinente.

De manera adicional este texto pretende examinar el proceso de evolución diacrónica de la tecnología de podcasting a lo largo de los últimos quince años. Dicho proceso se enmarca en el cambio conceptual de una realidad como internet, que identificamos con la web semántica manual o web 2.0, así como en el surgimiento y expansión de la figura del prosumidor de contenidos, con unas pautas de actuación bien diferenciadas respecto a las de las audiencias tradicionales.

Al mismo tiempo también se aspira a estudiar las posibilidades de difusión y distribución de todo tipo de contenidos, y de manera específica los referidos al patrimonio cultural, que proporcionan las distintas plataformas de podcasting que existen hoy en día en el mercado, centrándonos de manera especial en los casos de iVoox o SoundCloud, dos de las grandes referencias en el ámbito internacional.

\section{Estado de la cuestión}

El nuevo milenio conlleva, entre otras circunstancias, una notable mutación en la concepción de internet. Si hasta ese entonces se la identificaba con un gigantesco almacén de todo tipo de contenidos a partir de ese instante se la equipara con la denominada web semántica manual o web 2.0 en la terminología explicitada por DiNucci, quien considera que la red de redes supone una vía estratégica para mostrar cómo la web a la que estaban habituados los usuarios se transforma tanto en sus interfaces como en la visualización de sus contenidos ocasionando un profundo cambio en la experiencia de dichos usuarios y en sus procedimientos de interactuación ${ }^{5}$.

Esta idea inicial fue posteriormente conceptualizada por O'Reilly al definir esta novedosa internet como una mega ágora donde los internautas de todo el mundo establecen una charla a escala global en la que comparten conocimientos, inquietudes y experiencias, al tiempo que ese proceso colaborativo conforma un nuevo escenario de relación entre las corporaciones de toda índole y sus audiencias que marca un antes y un después en todo lo que atañe a la transmisión de contenidos $^{6}$.

\footnotetext{
${ }^{5}$ DI NUCCI, Darcy. Fragmented Future. Print Magazine [en línea]. 1999, vol. 53, n. 4, pp. 32, pp. 221 222. [Fecha de consulta: 23-01-2020]. Disponible en <http://darcyd.com/fragmented_future.pdf>.

6 O'REILLY, Tim. What is Web 2.0. Design Patterns and Business Models for the Next Generation of Software. O’Reilly [en línea]. 2005. [Fecha de consulta: 24-01-2020]. Disponible en
} 
Los trabajos de ambos investigadores fueron completados con numerosas aportaciones desde las más variadas perspectivas: esencialmente tecnológica, tanto en su faceta de investigación básica como aplicada, con los trabajos de Kamel Boulos y Wheeler ${ }^{7}$, Coleman y Levine $^{8}$, Bofil ${ }^{9}$ o Caruso10; en el ámbito de la educación y la formación, con las reflexiones de Andersen ${ }^{11}$, Grosseck $^{12}, \mathrm{McHaney}^{13}$ o Harris y Rea ${ }^{14}$; o en el campo de la comunicación, a partir de las propuestas de Briggs, Fumero y Roca, Cebrián Herreros o Mcnamara, entre otros.

Para Briggs esta novedosa noción de internet se identifica con sitios web convertidos en plataformas de computación que ofrecen sus aplicaciones a los usuarios finales ${ }^{15}$ al tiempo que Fumero y Roca consideran que deviene en un espacio con cabida para los agentes sociales ${ }^{16}$. Por su parte Cebrián Herreros enfatiza el hecho de que se asiste a un entorno emergente que apuesta por la interactividad de las audiencias ${ }^{17}$ mientras que Mcnamara centra su análisis en el rol que desempeñan como herramientas de dinamización de acciones de comunicación ${ }^{18}$.

\subsection{El peso específico de la interacción entre prosumidores y redes sociales en la consolidación del podcast}

Blogs, canales audiovisuales, portales de fotografía y música, plataformas

<http://www.oreillynet.com/pub/a/oreilly/tim/news/2005/09/30/what-is-web-20.html>.

${ }^{7}$ KAMEL BOULOS, Maged y WHEELER, Steve. The emerging Web 2.0 social software: an enabling suite os sociable technologies in health and health care education. Health Information and Libraries Journal [en línea]. 2007, vol. 24, pp. 2-23. [Fecha de consulta: 25-01-2020]. Disponible en <https://onlinelibrary.wiley.com/doi/full/10.1111/j.1471-1842.2007.00701.x>.

8 COLEMAN, David y LEVINE, Stewart. Collaboration 2.0: technology and best practices for successful collaboration in a Web 2.0 world. Silicon Valley: Happy About, 2008.

9 BOFIL, Leslie. Constructivism and Collaborating Using Web 2.0 Technology. Journal of Applied Learning Technology. 2013, vol. 3, n. 2, pp. 31-37.

10 CARUSO, Shirley. Towards Understanding the Role of Web 2.0 Technology in Self-Directed Learning And Job Performance. Contemporary Issues in Education Research [en línea]. 2018, vol. 11, n. 3, pp. 89-98. [Fecha de consulta: 23-01-2020]. Disponible en <https://clutejournals.com/index.php/CIER/article/view/10180/10254>.

11 ANDERSEN, Paul. What is Web 2.0? Ideas, technologies and implications for education. Bristol: JISC, 2007.

12 GROSSECK, Gabriela. To use or not to use Web 2.0 in higher education? Procedia Social and Behavioural Sciences [en línea]. 2009, vol. 1, n. 1, pp. 478-482. [Fecha de consulta: 27-01-2020]. Disponible en <https://www.sciencedirect.com/science/article/pii/S1877042809000895?via\%3Dihub>.

${ }^{13}$ MCHANEY, Roger. The New Digital Shoreline: How 2.0 and Milennials Are Revolutionizing Higher Education. Sterling: Stylus Publishing, 2012.

14 HARRIS, Albert y REA, Alan. Web 2.0 and Virtual World Technologies: A Growing Impact on IS Education. Journal of Information Systems Education [en línea]. 2019, vol. 20, n. 2, pp. 137-144. [Fecha de consulta: 03-01-2020]. Disponible $<$ https://aisel.aisnet.org/cgi/viewcontent.cgi?article=1271\&context=jise>.

${ }^{15}$ BRIGGS, Mark. Periodismo 2.0. Austin: Knight Foundation, 2007, p. 33.

${ }^{16}$ FUMERO, Antonio y ROCA, Genís. Web 2.0. Madrid: Fundación Orange, 2007, p. 10.

17 CEBRIÁN HERREROS, Mariano. Desarrollo del periodismo en Internet. Sevilla: Comunicación Social, 2010, p. 18.

18 MCNAMARA, Jim. Public communication practices in the Web 2.0-3.0 mediascape: The case of PRevolution. Prism [en línea]. 2013, vol. 7, n. 3, pp. 1-13. [Fecha de consulta: 29-01-2020]. Disponible en <https://www.prismjournal.org/uploads/1/2/5/6/125661607/v7-no3-a3.pdf>. 
educativas, redes sociales, servicios de sindicación de contenidos, wikis y podcasts, considerados tanto de forma individual como a través de las numerosas plataformas de distribución existentes, constituyen algunos de los elementos de esa emergente web 2.0 donde surge la figura del prosumidor como síntesis de productor y consumidor y en oposición a la tradicional consideración del receptor pasivo característico de la tradicional industria de los medios de comunicación de masas.

Esta noción de prosumidor es desarrollada por autores como Toffler, quien lo considera como un sujeto paradigmático de la tercera ola, entendida como un entorno que supera las habituales barreras geográficas características de las sociedades industrialmente avanzadas y auspicia el fenómeno de la globalización ${ }^{19}$, o Tapscott, que lo identifica con aquella persona que hace las cosas a su manera sin dejarse influir por las grandes corporaciones que deben adaptarse a este nuevo contexto ${ }^{20}$.

De esta manera, tal y como pone de manifiesto Bird, quedan establecidos un conjunto de procedimientos particularmente adaptados a las pautas de consumo globales y de comunicación e información de dichos prosumidores no sólo a la hora de llevar a cabo la distribución de los contenidos sino incluso en lo que se refiere a la propia generación de los mismos ${ }^{21}$, en el que una innovación como las redes sociales adquiere particular relevancia ${ }^{22}$.

Esa particular interacción entre prosumidores y redes sociales ha sido profusamente analizada en la literatura científica desde muy diversas perspectivas. Bellman entiende que esas redes sociales ya forman parte de la vida cotidiana de un relevante conjunto de ciudadanos que cada vez están más habituados al manejo de toda clase de dispositivos de carácter tecnológico que inciden sobre su devenir personal y profesional ${ }^{23}$.

19 TOFFLER, Alvin. The Third Wave. Nueva York: Bantam Books, 1980. 537 p. ISBN: 9780553144314.

20 TAPSCOTT, Don. The Digital Economy. Promise and Peril in The Age of Networked Intelligence. Nueva York: McGraw-Hill, 1995.

${ }^{21}$ BIRD, Elizabeth. Are We All Producers Now? Convergence and Media Audience Practices. Cultural Studies [en línea]. 2011, vol. 25, n. 4-5, pp. 502-516. [Fecha de consulta: 13-01-2020]. Disponible en $<$ https://www.tandfonline.com/doi/abs/10.1080/09502386.2011.600532>.

22 La base conceptual de estas novedosas plataformas se sitúa a finales de los años veinte del siglo XX con Karinthy y su teoría de los seis grados de separación, cuya formulación fue posteriormente recogida, ampliada y puesta al día por Watts (2003), quien subrayó cómo cualquier habitante de nuestro planeta se encuentra conectado a través de no más de cinco intermediarios o seis eslabones y de qué manera el número de individuos con quienes una persona se relaciona se incrementa conforme aumenta dicha cantidad de eslabones de la cadena.

Aunque a finales del siglo XX ya aparecen algunos ejemplos significativos de redes sociales online (con Classmate.com, 1995; AsianAvenue.com, 1997; y Sixdegrees.com, 1997, a la cabeza), no será hasta el comienzo del siglo XXI cuando se asista a su expansión y consolidación entre la comunidad internauta a partir de los ejemplos de Ryze.com (2001), Linkedln (2002), Tribe (2002), MySpace (2003), Xing (2003, inicialmente bajo el nombre OpenBC), Orkut (2004), Flickr (2004), Facebook (2004), YouTube (2005), Twitter (2006), Pinterest (2010) o Instagram (2010), entre otros particularmente significativos.

${ }^{23}$ BELLMAN, Barry. Computer Networks as Social Networks. Science [en línea]. 2001, vol. 293, n. 5537, pp. 2031-2034. [Fecha de consulta: 18-01-2020]. Disponible en <https://science.sciencemag.org/content/293/5537/2031>. 
A su vez Haythornthwaite subraya su profunda incidencia sobre la industria de los medios al otorgar a sus usuarios unas posibilidades de interactuación y generación de contenidos propios hasta entonces desconocidas ${ }^{24}$ mientras que Liben-Nowell y Kleinberg optan por enfatizar la faceta que tiene que ver con el alcance final de esos procesos de interacción y la formulación de un peculiar escenario de vínculos entre los individuos en el medio y largo plazo ${ }^{25}$.

Martos considera que "una red social es un conjunto de lazos entre diversos actores (personas físicas $u$ organizaciones), todos del mismo o similar tipo, que cuenta con un objetivo y unas normas; y cuyo uso se basa en ofrecer a los usuarios la posibilidad de narrar sus experiencias, incluso la propia biografía, explicar sus actividades, mostrar sus logros y describir sus intereses o ilusiones de forma o anónima o no"26.

Por su parte Bernal Bravo y Angulo Rasco, basándose en los trabajos de Boyd y Ellison ${ }^{27}$, las definen como "un servicio de Internet que permite a cualquier cibernauta construirse un perfil -público o semipúblico- dentro de un sistema gestionado por un tercero; compartir relaciones -experiencias, contenidos, etc.- con una lista de otros usuarios que, dependiendo de la privacidad autodefinida de su perfil, podrá tener acceso a todos los seguidores y a sus experiencias de los miembros de las listas que sigan las suyas" 28 .

Parra Valcarce y Martínez Arias ponen de relieve que en todas ellas existen determinados aspectos de coincidencia, entre los que destacan el uso de internet como plataforma de difusión de contenidos, el registro previo y la creación de un perfil para poder hacer uso del servicio, la interacción con otros usuarios, la generación de información que se comparte y la creación de grupos de personas con determinados vínculos específicos ${ }^{29}$.

A efectos prácticos, todo ello tiene como consecuencia un conjunto de nuevos procesos de socialización, cambios en la manera de interactuar corporaciones y audiencias o la inauguración de un espacio inédito de carácter virtual, con potencial

24 HAYTHORNTHWAITE, Caroline. Social networks and Internet connectivity effects. Information, Communication \& Society [en línea]. 2005, vol. 8, n. 2, pp. 125-147. [Fecha de consulta: 17-01-2020]. Disponible en <https://www.tandfonline.com/doi/abs/10.1080/13691180500146185>.

25 LIBEN-NOWELL, David y KLEINBERG, Jon. The link-prediction problem for social networks. Journal of the American Society for Information Science and Technology [en línea]. 2007, vol. 58, n. 7, pp. 1019-1031. [Fecha de consulta: 22-01-2020]. Disponible en $<$ https://onlinelibrary.wiley.com/doi/abs/10.1002/asi.20591>.

${ }^{26}$ MARTOS RUBIO, Ana. Redes Sociales. Madrid: Anaya Multimedia, 2010, p. 10.

27 BOYD, Danah y ELLISON, Nicole. Social Network Sites: Definition, History and Scholarship. Journal of Computer-mediated Comunication [en línea]. 2007, vol. 13, n. 1, pp. 210-230. [Fecha de consulta: 19-01-2020]. Disponible en <https://academic.oup.com/jcmc/article/13/1/210/4583062>.

28 BERNAL BRAVO, César y ANGULO RASCO, Félix. Interacciones de los jóvenes andaluces en las redes sociales. Comunicar [en línea]. 2012, vol. 40, p. 25. [Fecha de consulta: 17-01-2020]. Disponible

$<$ https://www.revistacomunicar.com/index.php?contenido=detalles\&numero=40\&articulo=40-201304>.

${ }^{29}$ PARRA VALCARCE, David y MARTíNEZ ARIAS, Santiago. Tecnologías de la Gestión Periodística de la Información Digital. Conceptos básicos. Madrid: Los Autores, 2018. 
impacto sobre la transmisión de normas, valores, actitudes y comportamientos personales y sociales en los términos expresados por García Galera y Del Hoyo Hurtado ${ }^{30}$, además de la entrada en un entorno singular apuntado por Islas Carmona donde se perciben unas muy distintas pautas de consumo por parte de los usuarios, entre las que adquieren creciente peso específico el in-forming, la colaboración o la búsqueda de respuestas que va mucho más allá de la tradicional actitud pasiva de los usuarios ante el poder de la industria de los medios de comunicación ${ }^{31}$.

Por consiguiente ese cambio conceptual de internet en combinación con los avances en el ámbito de las tecnologías de la información y las comunicaciones en facetas como el desarrollo de nuevas plataformas hardware y programas y aplicaciones software de la más variada índole o el diseño y lanzamiento al mercado de nuevos dispositivos portátiles entre los que tabletas electrónicas y teléfonos móviles inteligentes alcanzan particulares niveles de implantación sientan las bases para la popularización de los podcasts, tanto entre los proveedores de contenidos como entre sus audiencias.

\subsection{Evolución diacrónica de la herramienta podcast}

Como ya se indicó con anterioridad en 2004 surge la noción conceptual de podcast pese a que previamente existían iniciativas que podrían enmarcarse en este mismo tipo de planteamiento estratégico, sobre todo surgidas en el seno de determinadas corporaciones radiofónicas estadounidenses centradas en la emisión de contenidos musicales de tipo pop, rock y country, según subraya Berry ${ }^{32}$.

Desde el punto de vista de su evolución en el tiempo consideramos la existencia de cuatro etapas diferenciadas, a las que denominamos como experimental (finales del siglo XX a 2005), de consolidación (2006 a 2009), multimedia (2010 a 2013) y de eclosión (2014 hasta la actualidad) respectivamente. Con ligeras diferencias entre los diversos países tal realidad se percibe tanto a escala internacional en general como en el caso particular de los podcasts emitidos en lengua castellana en España y en Hispanoamérica, territorio donde se advierte la existencia de una avanzadilla de países como México, Colombia o Ecuador que contrasta con el retraso detectado en naciones como Guatemala o Venezuela conforme apuntan Blanco Hernández, Rodero Antón y Corredera de Colsa ${ }^{33}$.

30 GARCÍA GALERA, María del Carmen y HOYO HURTADO, Mercedes del. Redes sociales, un medio para la movilización juvenil. Zer Revista de Estudios de Comunicación [en línea]. 2013, vol. 18, n. 34, p. 114. [Fecha de consulta: 18-01-2020]. Disponible en <https://www.ehu.eus/ojs/index.php/Zer/article/view/10649/9887>.

31 ISLAS CARMONA, José Octavio. El prosumidor. El actor comunicativo de la sociedad de la ubicuidad. Palabra Clave [en línea]. 2008, vol. 11, n. 1, p. 37. [Fecha de consulta: 19-01-2020]. Disponible en <https://www.redalyc.org/pdf/649/64911103.pdf>.

32 BERRY, Richard. A Golden Age of Podcasting? Evaluation Serial in the Context of Podcast Histories. Journal of Radio \& Audio Media [en línea]. 2015, vol. 22, n. 2, pp. 170-178. [Fecha de consulta: 25-01-2020].

Disponible

en $<$ https://www.tandfonline.com/doi/abs/10.1080/19376529.2015.1083363>.

33 BLANCO HERNÁNDEZ, María; RODERO ANTÓN, Emma y CORREDERA DE COLSA, Luis Enrique. Evolución del conocimiento y consumo de podcasts en España e lberoamérica. Trípodos [en línea]. 2013, vol. 33, pp. 53-72. [Fecha de consulta: 17-01-2020]. Disponible en <http://www.tripodos.com/index.php/Facultat_Comunicacio_Blanquerna/article/view/97/277>.. 
La fase experimental arranca de finales de la última década del siglo $\mathrm{XX}$, cuando ni siquiera había quedado establecido el concepto de podcast. Tal realidad emerge en coincidencia con el fenómeno de la digitalización de la señal que soportaba los contenidos en línea y se sustenta a partir de un conjunto de innovaciones tecnológicas entre las que cabe destacar la ya referida sindicación de contenidos RSS y la generalización de un formato de compresión de ficheros de audio como es $\mathrm{MP}^{34}$.

En el caso de RSS marca la verdadera idiosincrasia de esta herramienta y la diferencia de los programas radiofónicos convencionales o incluso de los archivos sonoros colgados en las páginas web de una empresa periodística, al hacer posible la distribución masiva de contenidos en internet a través de determinados tipos de archivos especiales conforme apuntan Gutiérrez Porlán y Rodríguez Cifuentes ${ }^{35}$.

Con relación al formato MP3, Sterne subraya que ha quedado convertido en un verdadero estándar de facto para soportar contenidos de audio gracias a valores añadidos como una excelente relación entre calidad y volumen de información almacenada o no requerir de dispositivos portátiles dedicados para su reproducción ${ }^{36}$.

La segunda fase, de consolidación, comienza en la segunda mitad de la primera década del siglo XXI, al converger circunstancias como la expansión de la nueva generación de teléfonos móviles de tercera generación o smartphones, la creciente concienciación de las empresas informativas radiofónicas sobre las posibilidades expresivas del podcast y la cada vez más acusada capacidad de las audiencias a la hora de generar contenidos de audio posteriormente distribuidos a través de diversas plataformas.

En el caso de los teléfonos móviles de tercera generación, que gradualmente arrinconan los celulares convencionales pertenecientes a la etapa del GSM y tecnologías similares (como WAP, GPRS o incluso $i-M O D E$, entre otras ${ }^{37}$ ), se pone

\footnotetext{
${ }^{34}$ MP3 es un formato de compresión de audio cuyas bases estratégicas comienzan a conformarse a mediados de la década de los años ochenta del siglo XX con los trabajos y experimentaciones de un grupo de científicos que desarrollaban su actividad en el Fraunhofer-Gesellschaft de Alemania. Dichos estudios derivarían en lo que en la actualidad se entiende bajo tal denominación y que es conocido técnicamente como MPEG-1 capa 3. MPEG son las siglas correspondientes a Moving Picture Experts Group y aluden a un grupo de trabajo creado a principios de 1988 bajo la dirección de Leonardo Chiariglione cuyo objetivo fundamental era investigar los requerimientos precisos para la creación de un estándar para video digital en un soporte técnico. En sus investigaciones desarrollaron exclusivamente para el audio la "capa 3", siendo la aplicación de ésta en un equipo informático la que ha dado lugar a lo que hoy en día conocemos como MP3.

35 GUTIÉRREZ PORLÁN, Isabel y RODRÍGUEZ CIFUENTES, María Trinidad. Haciendo historia del podcast. Referencias sobre su origen y evolución. En: SOLANO FERNÁNDEZ, Isabel (ed.). Podcast educativo. Aplicaciones y orientaciones del m-learning para la enseñanza. Murcia: MAD Eduforma, 2010, pp. 17-32.

${ }^{36}$ STERNE, Jonathan. The mp3 as cultural artifact. New Media \& Society [en línea]. 2006, vol. 8, n. 5, pp. 825-842. [Fecha de consulta: 11-01-2020]. Disponible en: <https://journals.sagepub.com/doi/10.1177/1461444806067737>.

${ }^{37}$ GSM (Global System for Mobile Communication) fue un estándar de origen inicialmente europeo que se convirtió en el gran referente a escala internacional para la mayor parte de los usuarios y supuso el paso de la telefonía analógica de la primera generación a la digital. WAP (Wireless
} 
al servicio de las audiencias un dispositivo de cómodo acceso, alcance universal, muy fácil manejo y coste económico marginal muy bajo debido a su multifuncionalidad.

Al mismo tiempo llega el momento en que una parte significativa de las empresas informativas que desarrollan su actividad en el ámbito radiofónico perciben el podcast como una herramienta estratégica a tener en cuenta desde un doble punto de vista: por un lado, en lo que atañe a la búsqueda de nuevos segmentos de mercado sin necesidad de generar contenidos adicionales ni incurrir en cuantiosos costes económicos, concebidos a la manera esbozada por Ortiz Sobrino cuando pone de relieve que "los podcasts se han convertido en soporte publicitario y la comercialización de contenidos sonoros a través de pequeñas suscripciones o micropagos son prácticas que empiezan a normalizarse en algunos grupos radiofónicos ${ }^{38 " ;} \mathrm{y}$, por otra parte, en todo lo que tiene que ver con la búsqueda de nuevos formatos formales y de contenido que, según lo expuesto por González Conde, consiga "una nueva fórmula de programación fragmentada o de contenidos aislados, que sustituye la oferta de programas de emisión única y continuada y audición masiva ${ }^{39 " .}$

El último de los pilares sobre los que se sostiene este periodo de consolidación del podcast tiene que ver con ese cambio en el rol de los internautas al que se aludía con anterioridad. Las audiencias cuentan con una tecnología de muy fácil utilización y una más que notable capacidad de divulgación que optimiza su proceso de generación de ficheros de sonido. Como apuntan De Lara González y Del Campo Cañizares "el cambio más importante es que el usuario decide qué y cuándo ver/escuchar contenidos sin depender de la emisión en directo de los mismos, lo que conduce directamente a una especialización en la oferta" 40 .

Desde tal planteamiento Moreno Cazalla percibe dos factores críticos a la hora de explicar su creciente implantación: "la movilidad y las nuevas interfaces que poseen un elemento común, las pantallas; y la evolución de las aplicaciones móviles de podcatching, softwares que permiten a los usuarios acceder a una base de datos

\footnotetext{
Application Protocol) facilitó el acceso a contenidos de internet a través del teléfono móvil y abrió la senda que aúna interactividad y movilidad. GPRS (Global Packet Radio Service) sentó las bases para dar un salto hacia delante en capacidad de ancho de banda inalámbrico. i-MODE fue una tecnología de origen japonés, superior en prestaciones a las normativas anteriormente indicadas pero que implicaba la singularidad de unir en una misma compañía las figuras de operador, proveedor de acceso, proveedor de terminales, proveedor de servicios y agregador, un hecho impensable dentro del proceso de liberalización de las telecomunicaciones perceptible a nivel mundial.

38 ORTIZ SOBRINO, Miguel Ángel. Radio y post-radio en España: una cohabitación necesaria y posible. Área Abierta [en línea]. 2012, vol. 12, n. 2, p. 13. [Fecha de consulta: 10-01-2020]. Disponible en <https://revistas.ucm.es/index.php/ARAB/article/view/39637>.

39 GONZÁLEZ CONDE, Julia. La ciberradio. Nueva alternativa de futuro para la radio. Revista de Estudios de Juventud [en línea]. 2010, vol. 88, p. 53. [Fecha de consulta: 18-01-2020]. Disponible en $<$ http://www.injuve.es/sites/default/files/RJ88-05.pdf>.

40 LARA GONZÁLEZ, Alicia de y CAMPO CAÑIZARES, Elpidio del. El podcast como medio de divulgación científica y su capacidad para conectar con la audiencia. Revista Mediterránea de Comunicación [en línea]. 2018, vol. 9, n. 1, p. 351. [Fecha de consulta: 19-01-2020]. Disponible en $<$ https://www.mediterranea-comunicacion.org/article/view/2018-v9-n1-El-podcast-como-medio-dedivulgacion-cientifica-y-su-capacidad-para-conectar-con-la-audiencia>.
} 
de podcasts según categorías o por popularidad y que ofrecen servicios para predefinir los contenidos" 41 .

La tercera de las fases, multimedia, comienza propiamente en 2010 y supone la incorporación de podcasts no sólo en las páginas web de los cibermedios sino también en los portales de toda clase de organizaciones que comienzan a considerar su capacidad para servir de instrumento puesto al servicio de sus estrategias de comunicación corporativa, tanto en el ámbito externo (audiencias en general y/o accionistas en particular) como en el interno (plantilla propia, colaboradores y proveedores). Esta propuesta implica el acompañamiento de los elementos de sonido con textos, fotografías, infografías y diversos hipervínculos que completan su significado para esa clase de audiencias.

En tal sentido Barbeito Veloso y Perona Páez llaman la atención ante el hecho de que "las organizaciones no son ajenas a este fenómeno en crecimiento y, en su búsqueda constante de sistemas que propicien el diálogo y la interacción con sus stakeholders, han reparado en los beneficios que este tipo de productos pueden proporcionar en la construcción de sus marcas"42. Esta misma senda de análisis es seguida por Geets, quien considera que "los podcasts son una excelente forma de acceder a una nueva audiencia y de hacerles conocer una marca. La inversión suele ser mucho menor que la del vídeo y tiene la ventaja añadida de expandirse a un formato relativamente infravalorado, pero de rápido crecimiento: la marca de audio ${ }^{43}$.

A su vez Sellas Güell considera que se trata de un factor de naturaleza disruptiva cuyo empleo sistemático en esta faceta de la comunicación puede llegar a alterar las dinámicas entre organizaciones, profesionales y públicos una vez que se produzca su generalización, en un primer momento en las grandes corporaciones y con posterioridad en las de mediano y pequeño tamaño ${ }^{44}$.

Dentro del caso concreto de la industria de los medios de comunicación el gran impulso a esta fase multimedia viene de la mano de ProPublica, creada a finales de 2007 en Estados Unidos como entidad sin ánimo de lucro gracias al liderazgo del antiguo editor de The Wall Street Journal, Paul Steiger, y cuyos ingresos proceden de los fondos aportados por la Sandler Foundation y de donaciones privadas y la

\footnotetext{
41 MORENO CAZALLA, Lourdes. Podium podcast, cuando el podcast tiene acento español. Prisma Social [en línea]. 2017, vol. 18, p. 352. [Fecha de consulta: 22-01-2020]. Disponible en $<$ https://dialnet.unirioja.es/servlet/articulo?codigo $=6220235>$.

42 BARBEITO VELOSO, María Luz y PERONA PÁEZ, Juan José. Comunicación organizacional en clave sonora: el caso de Blink, los podcasts de BBVA. El Profesional de la Información [en línea]. 2019, vol. 28, n. 5, p. 2. [Fecha de consulta: 27-01-2020]. Disponible en $<$ <ttp://www.elprofesionaldelainformacion.com/contenidos/2019/sep/barbeito-perona.pdf $>$..

43 GEETS, Kevin. El éxito del 'podcast' y cómo tu marca puede aprovecharlo. MarketingNews [en línea]. 2019, 8 mayo. [Fecha de consulta: 27-01-2020]. Disponible en <https://www.marketingnews.es/tecnologia/opinion/1132179054405/exito-del-podcast-y-marca-puedeaprovecharlo.1.html>.

44 SELLAS GÜELL, Antoni. El uso del podcasting como herramienta de la identidad corporativa en la campaña del 90 aniversario de Iberia. El caso del podcast Aerolínea Momentos. Communication Papers [en línea]. 2018, vol. 7, n. 15, pp. 155-170. [Fecha de consulta: 22-01-2020]. Disponible en <https://revistes.udg.edu/communication-papers/article/view/22189>.
} 
gestión de la publicidad. Tal y como señala Parra Valcarce esta corporación periodística advirtió con rapidez las posibilidades multimedia del podcast, así como su capacidad de convertirse en un soporte narrativo con entidad propia, no necesariamente incardinado en una estructura específica sino como elemento independiente y con valor informativo autónomo ${ }^{45}$.

Cada podcast generado por el equipo de redacción de ProPublica dispone de un texto y de numerosos hipervínculos que permiten enriquecer su contenido y refuerzan dicha naturaleza autónoma, así como de distintos elementos gráficos estáticos como fotografías e infografías. Por término medio, su duración oscila entre los quince y los veinte minutos aunque no es infrecuente la aparición de casos excepcionales por su brevedad (incluso de dos tres minutos) o por su considerable extensión (por ejemplo una pieza generada en 2017 como The Breakthrough: What American Journalists Can Learn From Reporting Under Putin, supera la media hora de duración $)^{46}$.

A partir del año 2014 nos adentramos en la cuarta (y por el momento última) etapa de este proceso, caracterizada por la eclosión del podcast, tal y como ponen de relieve las cifras proporcionadas por diferentes análisis de mercado de la más variada índole. Corporaciones como Amazon, General Electric, Nintendo, Tinder o UBS a escala internacional o la entidad financiera BBVA en la esfera castellano parlante se encuentran en vanguardia a la hora de utilizarlos activamente. $Y$ algo similar sucede en el ámbito de las pequeñas y medianas empresas, de las asociaciones y fundaciones y de los internautas particulares.

En paralelo con dicha realidad se produce la aparición y/o consolidación de un considerable número de plataformas, tanto transnacionales como locales, concebidas para simplificar y optimizar el proceso de distribución de esta clase de tecnología. Ejemplos como Apple Podcasts, iVoox, SoundCloud, Spotify o Spreaker, entre otros muchos, resultan lo suficientemente significativos para comprender las tendencias de futuro en el corto y medio largo, así como las evidentes diferencias que se advierten con las plataformas de contenido de vídeos del estilo de YouTube o Vimeo.

Locker señala que una plataforma como iTunes de Apple registró un crecimiento en el número de descargas vinculadas con esta tecnología de 7.000 millones a 50.000 millones en el periodo que va de 2014 a $2018^{47}$. Por su parte Cortizo pone de manifiesto que en ese mismo lapso de tiempo el porcentaje de población de Estados Unidos que había escuchado en alguna ocasión un podcast pasó del treinta al

45 PARRA VALCARCE, David. Periodismo ciudadano y cambio social: análisis de iniciativas colaborativas en el ámbito del periodismo de investigación. Estudios sobre el Mensaje Periodístico [en línea]. 2017, vol. 23, n. 1, p. 142. [Fecha de consulta: 20-01-2020]. Disponible en $<$ https://revistas.ucm.es/index.php/ESMP/article/view/55587/50445>.

${ }^{46}$ Puede verse una extensa variedad de podcasts a través del subdominio de la página web de la compañía: https://www.propublica.org/podcast

47 LOCKER, Melissa. .Apple's podcast just topped 50 billion-all time downloads and streams. Fastcompany [en línea]. 2018, 25 de abril. [Fecha de consulta: 17-01-2020]. Disponible en $<$ https://www.fastcompany.com/40563318/apples-podcasts-just-topped-50-billion-all-time-downloadsand-streams>. 
cuarenta y cuatro por ciento y el número de consumidores habituales de esta tecnología (al menos uno al mes) se situaba por encima del veinticuatro por ciento ${ }^{48}$.

A su vez, un estudio elaborado por Edison Research para la compañía Triton Digital en 2017 permite una mejor comprensión cuantitativa y cualitativa de este fenómeno: el oyente medio de esta tecnología escucha en torno a las cinco horas semanales, más del setenta por ciento del consumo se produce a través de los teléfonos móviles inteligentes y cuatro de cada diez lo descarga en su dispositivo para escucharlo sin necesidad de una conexión a la red ${ }^{49}$.

Se advierte que el podcast es una tecnología con unos relevantes niveles de crecimiento potencial, susceptible de ser empleada en cualquier clase de sector productivo, válida para abordar cualquier temática con muy distintos tratamientos y adecuada para cualquier tipo de organización con independencia de su titularidad pública o privada, tamaño y capacidad financiera.

Para Shantikumar esta innovación abre un prometedor camino en la formación de profesionales sanitarios una vez que se haya expandido su influjo sobre los alumnos de estudios no universitarios ${ }^{50}$. Por su parte Chacón y Pérez explicitan que "El podcast constituye una herramienta mediadora para desarrollar aprendices autónomos de la lengua inglesa que, adicionalmente, refuerza en el estudiante el desarrollo de la confianza en sí mismo y la seguridad al momento de producir expresiones habladas en el idioma a aprender, es decir, reduce el estrés de tener que hablar en frente de sus compañeros al mismo tiempo que aumenta la motivación por su característica innovadora e interactiva" 51.

Kang y Gretzel aluden a que su utilización contribuye a optimizar la experiencia turística y mejora la sostenibilidad ambiental aplicada a este sector industrial ${ }^{52}$ mientras que Lotfian y Busso centran su atención en todo lo que tiene que ver con la mejora en los niveles de eficacia referidos a los procesos de comunicación afectiva considerados de una manera integrada ${ }^{53}$.

${ }^{48}$ CORTIZO, José Carlos. Podcasting, un mercado que está despegando a lo grande. En Digital [en línea]. 2018, 30 de mayo. [Fecha de consulta: 18-01-2020]. Disponible en $<$ https://en.digital/blog/podcasting-un-mercado-que-esta-despegando-a-lo-grande/854>.

49 Edison Research. The Podcast Consumer 2017 [en línea]. 2017. [Fecha de consulta: 24-01-2020]. Disponible en <http://www.edisonresearch.com/wp-content/uploads/2017/04/Podcast-Consumer2017.pdf>.

50 SHANTIKUMAR, Saran. From lecture theatre to portable media: students' perceptions of an enhanced podcast for revision. Medical Teacher [en línea]. 2009, vol. 31, n. 6, pp. 535-538. [Fecha de consulta: 29-01-2020]. Disponible

<https://www.tandfonline.com/doi/abs/10.1080/01421590802365584>.

51 CHACÓN, Carmen y PÉREZ, Clevia. El podcast como innovación en la enseñanza del inglés como lengua extranjera. Pixel-Bit Revista de Medios y Educación [en línea]. 2011, vol. 39, p. 44. [Fecha de consulta: 22-01-2020]. Disponible en <https://recyt.fecyt.es/index.php/pixel/article/view/61449/37462>. 52 KANG, Myunghwa y GRETZEL, Ulrike. Effects of podcast tours on tourist experiences in a national park. Tourism Management [en línea]. 2012, vol. 33, n. 2, pp. 440-455. [Fecha de consulta: 25-012020]. Disponible <https://www.sciencedirect.com/science/article/abs/pii/S0261517711001075?via\%3Dihub>.

53 LOFTIAN, Reza y BUSSO, Carlos. Building Naturalistic Emotionally Balanced Speech Corpus by Retrieving Emotional Speech from Existing Podcast Recordings. IEEE Transactions on Affective Computing [en línea]. 2019, vol. 10, n. 4, pp.471-483. [Fecha de consulta: 29-01-2020]. Disponible en 
En definitiva, todo hace prever que en el medio y largo plazo las perspectivas de desarrollo de los podcasts son sumamente prometedoras y que esta tecnología continuará con su trayectoria ascendente. Un reciente estudio de Kantar, referido a las previsiones de inversión publicitaria en la industria de los medios de comunicación correspondientes al año 2020 a escala internacional, subraya que el podcast será uno de los canales que más crecerá, apoyándose en factores como su naturalidad y cercanía que consiguen una audiencia muy receptiva y atractiva para los anunciantes y el todavía escaso grado de nivel de saturación de anuncios ${ }^{54}$.

\subsection{Surgimiento y consolidación de las plataformas de podcasting}

La interrelación entre las etapas de consolidación, multimedia y eclosión a las que aludíamos con anterioridad da como resultado la gradual creación de plataformas en línea destinadas a soportar los podcasts generados por esa creciente legión de autores corporativos y economías domésticas centrados en toda clase de temas. Como escribe López Villfranca "En 2014 la irrupción de Serial, un podcast cuyo objetivo era reabrir la investigación de un crimen ocurrido quince años atrás de una adolescente de origen surcoreano en Baltimore, marcó un antes y un después en el éxito del podcast radiofónico en Estados Unidos" 55 .

Su tipología es sumamente variada: desde las que permiten el alojamiento gratuito de contenidos hasta las que optan por el modelo de pago; las centradas en suscripciones y las que combinan éstas con la descarga libre de los programas; las especializadas frente a las de índole generalista; las que operan a una escala local por la idiosincrasia de sus contenidos (a pesar de la transnacionalidad inherente a internet) y las que desde el primer momento surgieron con planteamientos globales y de carácter internacional. En buena medida, todas ellas responden al desafío de afrontar la creciente fragmentación de audiencias conforme explicita Sullivan ${ }^{56}$.

Plataformas (y/o aplicaciones) como Apple Podcasts (i-Tunes), CastBox, iVoox, Live 365, Player FM, Podcast Go, Podcast Alley, Pocket Casts, Podfeed, Podomatic SoundCloud, Spotify, Spreaker o Tunelln, entre otras muchas, proporcionan hoy en día millones de programas de la más variada tipología a unas audiencias crecientes y ávidas de nuevos contenidos, al tiempo que permiten a distintos usuarios incorporar sus propios programas.

De esta manera se entra en una dinámica que no sólo facilita sino que incluso auspicia la disrupción en el mercado convencional de los contenidos de audio, sector

\footnotetext{
<https://ieeexplore.ieee.org/document/8003425>.

${ }^{54}$ Kantar. Media Trends \& Predictions 2020 [en línea]. 2019. 16 p. [Fecha de consulta: 30-01-2020]. Disponible en <https://kantar.turtl.co/story/media-trends-and-predictions-2020es/page/1?teaser=true>.

55 LÓPEZ VILLAFRANCA, Paloma. Estudio de casos de la ficción sonora en la radio pública, RNE, y en la plataforma de podcast del Grupo Prisa en España. Disertaciones [en línea]. 2019, vol. 12, n. 2, p. 68. [Fecha de consulta: 22-02-2020]. Disponible en <https://revistas.urosario.edu.co/index.php/disertaciones/article/view/6547/7299>

56 SULLIVAN, John L. The Platforms of Podcasting: Past and Present. Social Media \& Society [en línea]. 2019, vol. 5, n. 4, pp. 1-12. [Fecha de consulta: 28-01-2020]. Disponible en $<$ https://journals.sagepub.com/doi/pdf/10.1177/2056305119880002>.
} 
generalmente identificado con el de las empresas informativas radiofónicas, que es puesta de relieve por Newman y Gallo, quienes señalan el gradual crecimiento de estas plataformas y la inminente entrada en el mercado de competidores emergentes atraídos por las favorables expectativas de negocio ${ }^{57}$.

Conviene precisar que un caso distinto es el referido a las redes de podcasting, planteadas como la agrupación de diferentes productores de este tipo de contenidos que unen sus esfuerzos para una mejor distribución de los mismos. A diferencia de lo que sucede con las plataformas, esta clase de propuestas no permite la subida indiscriminada de programas sino que fomenta su unificación corporativa a partir de unos patrones operativos y logísticos previamente definidos.

Se trata de un planteamiento estratégico, consecuencia lógica del crecimiento de esta tecnología, que comienza en Estados Unidos a finales de la primera década del siglo XXI y que hasta la fecha está logrando resultados sumamente satisfactorios en este país por su capacidad para la generación de visitas y descargas de los podcasts. Productores como la National Public Radio, iHeartRadio o Wondery fueron capaces de superar los diez millones de visitantes únicos mensuales a sus contenidos de podcasting en enero de $2020^{58}$.

En lo que se refiere al mercado castellano parlante las primeras redes de podcasts propiamente dichas comienzan en 2014, con un proyecto como Posta surgido en Argentina, al que siguen otros como Podium Podcast y Cuonda en España o Dixo (en realidad ya fundado en 2005 aunque con una propuesta distinta a la actual) y Punto Primario en México como algunas de las referencias más significativas.

\section{Metodología}

\subsection{Selección de las plataformas de podcasting}

De todo el extenso conjunto de plataformas de podcasting existentes en el mercado hemos escogido dos para proceder al análisis del peso específico alcanzado por los contenidos temática y expresamente centrados en la difusión del patrimonio cultural dentro del entorno hispano parlante: iVoox y SoundCloud.

Son cinco los principales criterios para proceder a dicha selección: ser plataformas abiertas a todo tipo de usuarios productores de contenidos, más allá de su carácter corporativo o individual y de su titularidad pública y privada; extremada simplicidad a la hora de gestionar los diversos contenidos allí colgados; gratuidad en el proceso de incorporación de los programas; existencia significativa de podcasts que forman parte del acervo castellano parlante y que por consiguiente son accesibles en español; y disparidad de planteamientos conceptuales que permiten enriquecer la propuesta de análisis vía su contrastación y contraposición.

\footnotetext{
57 NEWMAN, Nic y GALLO, Nathan. News Podcasts and the Opportunities for Publishers. Oxford: Reuters Institute, 2019.

58 Podtrac. Podcast industry audience rankings [en línea]. 2020. [Fecha de consulta: 10-02-2020]. Disponible en <http://analytics.podtrac.com/industry-rankings/>.
} 


\subsubsection{La plataforma iVoox}

Creada en 2008 por el emprendedor español Juan Ignacio Solera, iVoox $^{59}$ es una plataforma que permite colgar, reproducir, descargar y compartir audios de todo tipo, incluyendo podcasts así como elementos del estilo de programas de radio convencionales, audiolibros o conferencias y seminarios, entre otros. En la actualidad cuenta alrededor de 500.000 podcasts y soporta en torno a los cincuenta millones de escuchas mensuales ${ }^{60}$.

Se trata de un servicio que no requiere ninguna clase de suscripción obligatoria, característica diferencial con otras plataformas similares que existen en este segmento y que acaso constituya una de las claves estratégicas que explique su considerable crecimiento, pese a que lo habitual y más operativo resulta proporcionar una serie de datos básicos, sobre todo en el caso de quienes aspiran a generar contenidos propios.

Entre sus diferentes funcionalidades figuran las referidas a la creación de listas personalizadas de podcasts en función de los criterios de búsqueda determinados por el usuario, suscripciones a determinados contenidos, selección semanal de los podcasts más destacados y posibilidad de formar parte de una comunidad de usuarios a través de la que seguir las tendencias de consumo, enviar mensajes a los restantes miembros o ver las recomendaciones y consejos de otros.

A lo largo de estos últimos años, coincidiendo con la etapa de eclosión de los podcasts anteriormente indicada, la plataforma ha efectuado un conjunto de movimientos estratégicos con el propósito de que la permitan consolidar su posición de negocio en un mercado cada vez más competitivo. Con el fin de optimizar el dispositivo prioritario de consumo, el smartphone, cuenta con sendas aplicaciones móviles para los sistemas operativos iOS de Apple y Android.

En 2018 lanzó una plataforma que permite la monetización de determinados contenidos generados por los productores mediante un programa de suscripción de carácter mensual que otorga a las audiencias la opción de realizar una aportación de carácter voluntario y variable. Esta plataforma se completó con la funcionalidad iVoox Plus, que integra un sistema de micromecenazgo. En esta misma línea en 2019 puso en marcha la funcionalidad iVoox Originals, con la que, en palabras de su fundador, "pretende evitar uno de los males del mundo del podcast, la multisindicación" 61 .

Gracias a todo ello en la actualidad se ha consolidado como la principal plataforma de podcasts generados y distribuidos en español, contribuyendo de manera significativa en el ámbito castellano parlante a una ruptura de las dinámicas

\footnotetext{
${ }^{59}$ La página web de la plataforma iVoox es $<$ https://www.ivoox.com/ $>$.

60 VILLANUEVA, Xavi. Qué es iVoox con Juan Ignacio Solera. Abismo FM [en línea]. 2019. [Fecha de consulta: 30-01-2020]. Disponible en <https://abismofm.com/que-es-ivoox/>.

${ }^{61}$ Entrevista a Juan Ignacio Solera, fundador de iVoox, publicada en el ciberperiódico El Diario el 15 de noviembre de 2019. [Fecha de consulta: 31-01-2020]. Disponible en <https://www.eldiario.es/aragon/cultura/podcast-fecha-formato-buscan-difundir_0_963454574.html>.
} 
narrativas de toda clase de contenidos, tanto en su configuración transmedia como en las estrategias multiplataforma, según señalan García Marín y Aparici Marino62.

\subsubsection{La plataforma SoundCloud}

SoundCloud $d^{63}$ es una plataforma creada en Suecia en 2007 por Alex Ljung y Eric Wahlforss. Aunque en sus comienzos se orientó con una red social para músicos, conforme a los deseos de sus fundadores, con el tiempo evolucionó hacia una plataforma de podcasting similar a las del resto del mercado, alcanzando a finales de 2019 la cifra de 200 millones de usuarios.

Para optimizar el alojamiento de esta innovación puso en marcha una funcionalidad adicional denominada Podcasting en SoundCloud y optó por un sistema que requería la creación de una cuenta de usuario que tenía la doble misión de servir como perfil de usuario y enlazarlo con sus diferentes redes sociales o incluso con su propia página web en caso de disponer de ella.

De acuerdo con esta estrategia de diversificación el servicio orientó una parte apreciable de sus contenidos al alojamiento de podcasts de carácter educativo dirigidos tanto al nivel preuniversitario como al universitario. Para alcanzar dicho propósito se optó por la firma de diferentes acuerdos de colaboración con entidades formativas, así como por la puesta en marcha de una aplicación móvil y de distintas funcionalidades especialmente concebidas para dicho ámbito, entre las que Viñas destacaba la referida a comentarios cronometrados ${ }^{64}$.

Esta faceta pedagógica de la plataforma es retomada en las investigaciones de autoras como Birch, quien considera que estas potencialidades resultan particularmente relevantes en el ámbito específico de la enseñanza de contenidos musicales a través de teléfonos celulares vía su aplicación móvil65, así como de Grau Ibarra, para la que "SoundCloud puede contribuir en la distribución de los podcast educativos de la UNED para apoyar los procesos de enseñanzaaprendizaje"66.

62 GARCÍA MARÍN, David y APARICI MARINO, Roberto. Nueva comunicación sonora. Cartografía, gramática y narrativa transmedia del podcasting. El Profesional de la Información [en línea]. 2018, vol. 27, n. 5, pp. 1071-1081. [Fecha de consulta: 26-01-2020]. Disponible en $<$ http://www.elprofesionaldelainformacion.com/contenidos/2018/sep/11.html>.

${ }^{63}$ La página web de la plataforma SoundCloud es $<$ https://soundcloud.com/ $>$, y el subdominio donde se encuentran sus contenidos de podcasting <https://creators.soundcloud.com/podcasting $>$.

64 VIÑAS, Meritxell. SoundCloud: El audio como como potente herramienta en educación. Totemguard [en línea]. 2012. [Fecha de consulta: 28-01-2020]. Disponible en <https://www.totemguard.com/aulatotem/2012/06/soundcloud-el-audio-como-potente-herramienta-eneducacion/>.

${ }^{65} \mathrm{BIRCH}$, Heather. Potential of SoundCloud for mobile learning in music education: a pilot study. International Journal of Mobile Learning and Organisation [en línea]. 2017, vol. 11, n. 1, pp. 30-40. [Fecha de consulta: 31-01-2020]. Disponible en <https://www.researchgate.net/profile/Heather_Birch3/publication/312011543_Potential_of_SoundClou d_for_mobile_learning_in_music_education_A_pilot_study/links/5c7e9599458515831f8567̄ea/Potenti al-of-SoundCloud-for-mobile-learning-in-music-education-A-pilot-study.pdf>..

66 GRAU IBARRA, Katia. SoundCloud: sus posibilidades y limitaciones como una plataforma de distribución de podcast educativos. Revista de Educación a Distancia [en línea]. 2019, vol. 59, p. 13. [Fecha de consulta: 30-01-2020]. Disponible en 
Dentro de su apuesta por encontrar nuevas fórmulas que permitan su crecimiento a comienzos de 2019 la plataforma lanzó el servicio SoundCloud Premier dirigido a creadores de contenidos musicales y meses después permitió la introducción de publicidad en el segmento del mercado de contenidos castellano parlantes, hecho que coincidió en el tiempo con la implantación de una funcionalidad de pago denominada SoundCloud Go+ que ofrecía un extenso catálogo musical a los suscriptores.

Hoy en día, tras distintos altibajos y rumores sobre su compra por parte de terceras compañías, la plataforma se ha erigido en uno de los principales actores del mercado del podcasting globalmente considerado (y no únicamente del centrado en los contenidos musicales), ampliando de manera continuada el número de prestaciones.

\subsection{Ficha de análisis y determinación de podcasts}

Con el fin de determinar cuál es el peso específico de los podcasts centrados temática y expresamente en la difusión del patrimonio cultural dentro del entorno castellano parlante se ha procedido en primer lugar a elaborar una ficha de análisis que considera los siguientes aspectos: denominación, descripción, URL de localización, entidad productora, fecha de comienzo del podcast, número de programas acumulado hasta la fecha (cerrándose el análisis el 15 de febrero de 2020), nivel de actualización de los contenidos generados, duración de los podcasts (mínima, máxima y promedio) y estructura de contenidos.

Esta ficha no incluye las referencias a la cantidad de visitas y/o descargas generadas por cada uno de los podcast dado que el actual sistema de medición de ambas plataformas no permite la obtención de datos suficientemente verificados y contrastados.

A continuación se ha procedido a la búsqueda de los diferentes podcasts incorporados en las plataformas iVoox y SoundCloud centrados en dichos contenidos.

En el caso de iVoox se han encontrado un total de nueve podcasts integrados en sendos canales referidos a la cuestión, correspondientes a:

- Patrimonio Arqueológico, dentro del canal Patrimonio Cultural.

- Seminario Internacional de Reciclaje de Edificaciones, dentro del canal Instituto Distrital de Patrimonio Cultural.

- Patrimonio Cultural del Paraguay, dentro del canal del mismo nombre.

- Asociación en Defensa del Patrimonio Cultural Natural, dentro del canal del mismo nombre.

- Paisaje Cultural, dentro del canal Podcast Patrimonial.

- Vive tu Patrimonio, dentro del canal del mismo nombre.

- Arquisabios, el Podcast, Caminando la Ciudad, dentro del canal del mismo 
nombre.

- Radio GPC, dentro del canal del mismo nombre.

- Patrimonio Cultural y Artístico de Diputación, dentro del canal del mismo nombre.

En lo que se refiere a SoundCloud se ha determinado la existencia de tres podcasts que cumplen con tales características y cuyas respectivas denominaciones son las siguientes:

- ONG Coprous.

- Geiser Martín.

- Patrimonio Cultural.

Debe aclararse que a diferencia de lo que sucede con iVoox, en esta plataforma los podcasts no se vinculan con un canal (esta diferencia se advierte en el criterio referido a la denominación que aparece en primer lugar en cada una de las fichas de referencia).

En nuestra investigación sólo se consideran los podcasts específicamente generados, no los contenidos procedentes de programas radiofónicos convencionales que posteriormente son colgados en la plataforma online o la grabación ad hoc de determinados eventos e iniciativas de índole cultural o canales de carácter generalista que únicamente contemplan la cuestión de una manera tangencial.

De ahí que se hayan descartado los podcasts:

- Radio Teror (Gran Canaria, España).

- Patrimonio en la Onda (Zamora, España).

- Encuentro UNIA (Jaén, España).

- Museos en Vivo (Medellín, Colombia).

- Radio Don Matías (Santiago, Chile).

- Por el Veto Presidencial Ley de Patrimonio Cultural (Tabuzcalco, México).

- Escalinata (La Habana, Cuba).

- Suroeste defiende su patrimonio natural y cultural (Antioquía, Colombia), o

- Leyendas Patrimonio Cultural Intangible (Hidalgo, México) dentro de la plataforma iVoox.

Por motivos de similar índole han quedado descartados los podcasts:

- Unschvirtual (Ayachucho, Perú).

- Ministerio de la Cultura (Caracas, Venezuela) y,

- Audiovisuales Podcast UNED (Madrid, España), integrados en SoundCloud. 


\section{Resultados de la investigación}

\subsection{Plataforma iVoox}

\begin{tabular}{|c|c|}
\hline \multicolumn{2}{|c|}{ Podcast Patrimonio Arqueológico (Canal Patrimonio Cultural) } \\
\hline Denominación & $\begin{array}{l}\text { Patrimonio Arqueológico. Se encuentra dentro del canal Patrimonio } \\
\text { Cultural }\end{array}$ \\
\hline Descripción & $\begin{array}{l}\text { Centrado en el análisis del patrimonio arqueológico y rupestre en } \\
\text { Colombia }\end{array}$ \\
\hline URL de localización & $\begin{array}{l}\text { https://www.ivoox.com/audios-patrimonio- } \\
\text { cultural_s0_f2212033_p2_1.html?o=all }\end{array}$ \\
\hline Entidad productora & $\begin{array}{l}\text { Rupestre Web (http://www.rupestreweb.info/), una publicación } \\
\text { electrónica especializada en la investigación del arte rupestre en el } \\
\text { área de América Latina }\end{array}$ \\
\hline Fecha de comienzo & 7 de mayo de 2016 \\
\hline $\begin{array}{l}\text { Número } \\
\text { programas } \\
\text { acumulados }\end{array}$ & 9 \\
\hline $\begin{array}{l}\text { Nivel } \\
\text { actualización } \\
\text { contenidos }\end{array}$ & El último podcast corresponde al 6 de marzo de 2018 \\
\hline Duración & $\begin{array}{l}\text { Mínima: } 9 \text { minutos } 51 \text { segundos } \\
\text { Máxima: } 3 \text { horas } 54 \text { minutos } 40 \text { segundos } \\
\text { Promedio aproximado: por encima de } 1 \text { hora } 30 \text { minutos }\end{array}$ \\
\hline $\begin{array}{l}\text { Estructura } \\
\text { contenidos }\end{array}$ & $\begin{array}{l}\text { Cada uno de los podcasts es de carácter temático: entrevistas, } \\
\text { conferencias y jornadas de investigación }\end{array}$ \\
\hline
\end{tabular}

Fig. 1: Podcast Patrimonio Arqueológico (Canal Patrimonio Cultural). Fuente: Elaboración propia.

\begin{tabular}{|l|l|}
\hline \multicolumn{2}{|c|}{ Podcast Seminario Internacional de Reciclaje de Edificaciones (Canal Instituto } \\
Distrital de Patrimonio Cultural)
\end{tabular}


David PARRA VALCARCE y Charo ONIEVA MALLERO. El uso del podcast para la difusión del patrimonio cultural en el entorno hispanoparlante: análisis de las plataformas iVoox y SoundCloud.

contenidos presentadas durante el transcurso de este seminario internacional

Fig. 2: Podcast Seminario Internacional de Reciclaje de Edificaciones (Canal Instituto Distrital de Patrimonio Cultural). Fuente: Elaboración propia.

\begin{tabular}{|c|c|}
\hline \multicolumn{2}{|c|}{ Podcast Patrimonio Cultural del Paraguay (Canal Patrimonio Cultural del Paraguay) } \\
\hline Denominación & $\begin{array}{l}\text { Patrimonio Cultural del Paraguay. Se encuentra dentro del canal } \\
\text { Patrimonio Cultural del Paraquay }\end{array}$ \\
\hline Descripción & Dedicado a la historia de Paraguay \\
\hline $\begin{array}{l}\text { URL } \\
\text { localización }\end{array}$ & $\begin{array}{l}\text { https://www.ivoox.com/audios-patrimonio-cultural-del- } \\
\text { paraguay_s0_f2189608_p2_1.html?o=all }\end{array}$ \\
\hline Entidad productora & $\begin{array}{l}\text { Aranduvera (http://aranduvera.com.py/), una biblioteca digital abierta } \\
\text { creada en } 2014 \text { centrada en la preservación digital del acervo } \\
\text { bibliográfico histórico de Paraguay }\end{array}$ \\
\hline Fecha de comienzo & 12 de septiembre de 2015 \\
\hline $\begin{array}{l}\text { Número } \\
\text { programas } \\
\text { acumulados }\end{array}$ & 1 \\
\hline $\begin{array}{l}\text { Nivel } \\
\text { actualización } \\
\text { contenidos }\end{array}$ & No existe al haber un único podcast \\
\hline Duración & $\begin{array}{l}\text { Mínima: } 3 \text { minutos } 25 \text { segundos } \\
\text { Máxima: } 3 \text { minutos } 25 \text { segundos } \\
\text { Promedio aproximado: } 3 \text { minutos } 25 \text { segundos }\end{array}$ \\
\hline $\begin{array}{l}\text { Estructura } \\
\text { contenidos }\end{array}$ & $\begin{array}{l}\text { Texto que relata fechas de relevancia que atañen a las mujeres de } \\
\text { Paraguay }\end{array}$ \\
\hline
\end{tabular}

Fig. 3: Podcast Patrimonio Cultural del Paraguay (Canal Patrimonio Cultural del Paraguay). Fuente: Elaboración propia.

\begin{tabular}{|l|l|}
\hline \multicolumn{2}{|c|}{ Podcast Asociación en Defensa del Patrimonio Cultural Natural (Canal Asociación en } \\
Defensa del Patrimonio Cultural Natural)
\end{tabular}




\begin{tabular}{|l|l|l|l|l}
$\begin{array}{l}\text { Estructura de } \\
\text { contenidos }\end{array}$ & $\begin{array}{l}\text { Cada podcast se centra en un aspecto temático concreto. Incluye } \\
\text { entrevistas, noticias y reportajes }\end{array}$ \\
\hline
\end{tabular}

Fig. 4: Podcast Asociación en Defensa del Patrimonio Cultural Natural (Canal Asociación en Defensa del Patrimonio Cultural Natural). Fuente: Elaboración propia.

\begin{tabular}{|l|l|}
\hline \multicolumn{2}{|c|}{ Podcast Paisaje Cultural (Canal Podcast Patrimonial) } \\
\hline Denominación & $\begin{array}{l}\text { Paisaje Cultural. Se encuentra dentro del canal Podcast } \\
\text { Patrimonial }\end{array}$ \\
\hline Descripción & Centrado en la divulgación y difusión del patrimonio de Bolivia \\
\hline URL de localización & $\begin{array}{l}\text { https://www.ivoox.com/podcast-podcast- } \\
\text { patrimonial_sq_f1851118_1.html }\end{array}$ \\
\hline Entidad productora & $\begin{array}{l}\text { Sra. Patrimonio, podcaster de carácter individual que dispone de } \\
\text { una cuenta en Twitter (https://twitter.com/es_patrimonio?s=20) }\end{array}$ \\
\hline $\begin{array}{l}\text { Fecha de comienzo } \\
\text { Número de } \\
\text { programas } \\
\text { acumulados }\end{array}$ & 1 \\
\hline $\begin{array}{l}\text { Nivel debrero de 2020 } \\
\text { actualización } \\
\text { contenidos }\end{array} \quad$ de & Imposible de determinar al existir un único programa \\
\hline $\begin{array}{l}\text { Duración } \\
\text { de }\end{array}$ & $\begin{array}{l}\text { Mínima: 1 hora 10 minutos 13 segundos } \\
\text { Máxima: 1 hora 10 minutos 13 segundos } \\
\text { Promedio aproximado: 1 hora 10 minutos 13 segundos }\end{array}$ \\
\hline $\begin{array}{l}\text { Estructura } \\
\text { contenidos }\end{array}$ & $\begin{array}{l}\text { Se plantea en forma de dialogo alrededor de una mesa de } \\
\text { desayuno }\end{array}$ \\
\hline
\end{tabular}

Fig. 5: Podcast Paisaje Cultural (Canal Podcast Patrimonial). Fuente: Elaboración propia.

\begin{tabular}{|c|c|}
\hline \multicolumn{2}{|c|}{ Podcast Vive tu Patrimonio (Canal Vive tu Patrimonio) } \\
\hline Denominación & $\begin{array}{l}\text { Vive tu Patrimonio. Se encuentra dentro del canal Vive tu } \\
\text { Patrimonio }\end{array}$ \\
\hline Descripción & $\begin{array}{l}\text { Centrado en la difusión y discusión del patrimonio cultural en } \\
\text { diferentes países }\end{array}$ \\
\hline URL de localización & $\begin{array}{l}\text { https://www.ivoox.com/podcast-vive-tu- } \\
\text { patrimonio sq f1735492 1.html }\end{array}$ \\
\hline Entidad productora & $\begin{array}{l}\text { Julio Sánchez (http://vivetupatrimonio.com/), un podcaster particular } \\
\text { que forma parte de la empresa Muro Promotora y Consultora } \\
\text { establecida en Perú }\end{array}$ \\
\hline Fecha de comienzo & 15 de julio de 2019 \\
\hline $\begin{array}{l}\text { Número } \\
\text { programas } \\
\text { acumulados }\end{array}$ & 7 \\
\hline $\begin{array}{l}\text { Nivel } \\
\text { actualización } \\
\text { contenidos }\end{array}$ & El último podcast está fechado el 3 de noviembre de 2019 \\
\hline Duración & $\begin{array}{l}\text { Mínima: } 28 \text { minutos } 11 \text { segundos } \\
\text { Máxima: } 1 \text { hora } 54 \text { minutos } 44 \text { segundos } \\
\text { Promedio aproximado: } 50 \text { minutos }\end{array}$ \\
\hline $\begin{array}{l}\text { Estructura } \\
\text { contenidos }\end{array}$ & Cada podcast incorpora una mesa redonda o una conferencia \\
\hline
\end{tabular}

Fig. 6: Podcast Vive tu Patrimonio (Canal Vive tu Patrimonio). Fuente: Elaboración propia. 
David PARRA VALCARCE y Charo ONIEVA MALLERO. El uso del podcast para la difusión del patrimonio cultural en el entorno hispanoparlante: análisis de las plataformas iVoox y SoundCloud.

\begin{tabular}{|c|c|}
\hline \multicolumn{2}{|c|}{$\begin{array}{l}\text { Podcast Arquisabios, el Podcast, Caminando la Ciudad (Canal Arquisabios, el } \\
\text { Podcast, Caminando la Ciudad) }\end{array}$} \\
\hline Denominación & $\begin{array}{l}\text { Arquisabios, el Podcast, Caminando la Ciudad. Se encuentra } \\
\text { dentro del canal Arquisabios, el Podcast, Caminando la Ciudad }\end{array}$ \\
\hline Descripción & $\begin{array}{l}\text { Centrado en la concienciación de la relevancia del patrimonio } \\
\text { arquitectónico y el cuidado de los adultos mayores }\end{array}$ \\
\hline URL de localización & $\begin{array}{l}\text { https://www.ivoox.com/podcast-arquisabios-el-podcast-caminando- } \\
\text { la-ciudad_sq_f1711001_1.html }\end{array}$ \\
\hline Entidad productora & Verónica Zambrano, una podcaster particular \\
\hline Fecha de comienzo & 23 de mayo de 2019 \\
\hline $\begin{array}{ll}\text { Número } & \text { de } \\
\text { programas } & \\
\text { acumulados } & \\
\end{array}$ & 2 \\
\hline $\begin{array}{l}\text { Nivel } \\
\text { actualización } \\
\text { contenidos }\end{array}$ & $\begin{array}{l}\text { El segundo y último de los podcasts está fechado el } 7 \text { de } \\
\text { noviembre de } 2019\end{array}$ \\
\hline Duración & $\begin{array}{l}\text { Mínima: } 9 \text { minutos } 53 \text { segundos } \\
\text { Máxima: } 17 \text { minutos } 48 \text { segundos } \\
\text { Promedio aproximado: } 13 \text { minutos }\end{array}$ \\
\hline $\begin{array}{l}\text { Estructura } \\
\text { contenidos }\end{array}$ & $\begin{array}{l}\text { Dos locutores recrean los contenidos planteados a partir de la } \\
\text { intervención de dos personajes de ficción: la viajera en el tiempo } \\
\text { Arquiza y el abuelo Cano }\end{array}$ \\
\hline
\end{tabular}

Fig. 7: Podcast Arquisabios, el Podcast, Caminando la Ciudad (Canal Arquisabios, el Podcast, Caminando la Ciudad). Fuente: Elaboración propia.

\begin{tabular}{|c|c|}
\hline \multicolumn{2}{|r|}{ Podcast Radio GPC (Canal Radio GPC) } \\
\hline Denominación & Radio GPC. Se encuentra dentro del canal Radio GPC \\
\hline Descripción & Análisis del estado del patrimonio cultural español \\
\hline $\begin{array}{l}\text { URL de } \\
\text { localización }\end{array}$ & https://www.ivoox.com/podcast-radio-gpc_sq_f1851311_1.html \\
\hline $\begin{array}{l}\text { Entidad } \\
\text { productora }\end{array}$ & $\begin{array}{l}\text { Radio GPC, cuyos contenidos se centran en el análisis del patrimonio } \\
\text { español. Es impulsado por el grupo de investigación sobre gestión del } \\
\text { patrimonio cultural de la Universidad Complutense de Madrid } \\
\text { (https://www.ucm.es/gpc/) }\end{array}$ \\
\hline $\begin{array}{l}\text { Fecha } \\
\text { comienzo }\end{array}$ & 6 de febrero de 2020 \\
\hline $\begin{array}{l}\text { Número } \\
\text { programas }\end{array}$ & 2 \\
\hline $\begin{array}{ll}\text { Nivel } & \text { de } \\
\text { actualización } & \text { de } \\
\text { contenidos } & \end{array}$ & El último podcast lleva fecha de 14 de febrero de 2020 \\
\hline Duración & $\begin{array}{l}\text { Mínima: } 25 \text { minutos } 57 \text { segundos } \\
\text { Máxima: } 33 \text { minutos } 3 \text { segundos } \\
\text { Promedio aproximado: } 30 \text { minutos }\end{array}$ \\
\hline $\begin{array}{l}\text { Estructura de } \\
\text { contenidos }\end{array}$ & $\begin{array}{l}\text { Se entremezclan entrevistas con especialistas en la materia y noticias } \\
\text { de actualidad }\end{array}$ \\
\hline
\end{tabular}

Fig. 8: Podcast Radio GPC (Canal Radio GPC). Fuente: Elaboración propia. 


\begin{tabular}{|l|l|}
\hline \multicolumn{2}{|c|}{ Podcast Patrimonio Cultural y Artístico de Diputación (Canal Patrimonio Cultural y } \\
Artístico de Diputación)
\end{tabular}

Fig. 9: Podcast Patrimonio Cultural y Artístico de Diputación (Canal Patrimonio Cultural y Artístico de Diputación). Fuente: Elaboración propia.

\subsection{Plataforma SoundCloud}

\begin{tabular}{|l|l|}
\hline \multicolumn{2}{|c|}{ Podcast ONG Coprous } \\
\hline Denominación & ONG Coprous \\
\hline Descripción & Análisis sobre la gestión del patrimonio y las artes en general \\
\hline URL de localización & https://soundcloud.com/user-376660698 \\
\hline Entidad productora & $\begin{array}{l}\text { Fundación para la Cooperación y el Progreso de Usiacurí } \\
\text { (https://www.facebook.com/coprous/), una entidad no gubernamental } \\
\text { de esta localidad de Colombia }\end{array}$ \\
\hline $\begin{array}{l}\text { Fecha de comienzo } \\
\text { Número de } \\
\text { programas } \\
\text { acumulados }\end{array}$ & 12 \\
\hline $\begin{array}{l}\text { Nivel } \\
\text { actualización } \\
\text { contenidos }\end{array} \quad$ de & $\begin{array}{l}15 \text { de febrero de 2020. Máximo nivel de actualización de esta } \\
\text { iniciativa novedosa }\end{array}$ \\
\hline $\begin{array}{l}\text { Duración } \\
\text { Estructura }\end{array}$ & $\begin{array}{l}\text { Mínima: 2 minutos 8 segundos } \\
\text { Máxima: 15 minutos 1 segundo } \\
\text { Promedio aproximado: 12 minutos }\end{array}$ \\
\hline dentenidos & $\begin{array}{l}\text { Mezcla de contenidos didácticos e informativos que incluye noticias } \\
\text { de actualidad y entrevistas con especialistas }\end{array}$ \\
\hline
\end{tabular}

Fig. 10: Podcast ONG Coprous. Fuente: Elaboración propia.

\begin{tabular}{|l|l|}
\hline \multicolumn{2}{|c|}{ Podcast Geiser Martín } \\
\hline Denominación & Geiser Martín \\
\hline Descripción & Conservación y difusión del patrimonio cultural y medioambiental \\
\hline URL de & https://soundcloud.com/geiser-martin-medina \\
\hline
\end{tabular}


David PARRA VALCARCE y Charo ONIEVA MALLERO. El uso del podcast para la difusión del patrimonio cultural en el entorno hispanoparlante: análisis de las plataformas iVoox y SoundCloud.

\begin{tabular}{|l|l|}
\hline $\begin{array}{l}\text { localización } \\
\text { Entidad } \\
\text { productora }\end{array}$ & $\begin{array}{l}\text { Geiser Gerardo Martín Medina, podcaster particular que forma parte del } \\
\text { Instituto Nacional de Antropología de } \\
\text { (https://inah.academia.edu/GeiserMart\%C3\%ADnMedina) }\end{array}$ \\
\hline $\begin{array}{l}\text { Fecha de } \\
\text { comienzo }\end{array}$ & 26 de agosto de 2018 \\
\hline $\begin{array}{l}\text { Número de } \\
\text { programas } \\
\text { acumulados }\end{array}$ & 2 \\
\hline $\begin{array}{l}\text { Nivel de } \\
\text { actualización de } \\
\text { contenidos }\end{array}$ & $\begin{array}{l}\text { El último podcast se colgó en 2019 (la plataforma no especifica mes ni } \\
\text { día) }\end{array}$ \\
\hline $\begin{array}{l}\text { Duración } \\
\text { Mínima: 24 minutos 2 segundos } \\
\text { Máxima: } 56 \text { minutos 18 segundos } \\
\text { Promedio aproximado: 40 minutos }\end{array}$ \\
\hline contenidos de & Incluye entrevistas y reportajes sobre la cuestión \\
\hline
\end{tabular}

Fig. 11: Podcast Geiser Martín. Fuente: Elaboración propia.

\begin{tabular}{|l|l|}
\hline \multicolumn{2}{|l|}{ Podcast Patrimonio Cultural } \\
\hline Denominación & Patrimonio Cultural \\
\hline Descripción & $\begin{array}{l}\text { Centrado en la difusión y rescate del patrimonio cultural de El } \\
\text { Salvador }\end{array}$ \\
\hline URL de localización & https://soundcloud.com/patrimonio-c \\
\hline Entidad productora & $\begin{array}{l}\text { Patrimonio Cultural, revista cultural producida en la localidad } \\
\text { de Santa Ana de El Salvador }\end{array}$ \\
\hline Fecha de comienzo & 2014 (la plataforma no especifica mes ni día) \\
\hline $\begin{array}{l}\text { Número de programas } \\
\text { acumulados }\end{array}$ & 1 \\
\hline $\begin{array}{l}\text { Nivel de actualización de } \\
\text { contenidos }\end{array}$ & 2014 (la plataforma no especifica mes ni día) \\
\hline Duración & $\begin{array}{l}\text { Mínima: 36 minutos 33 segundos } \\
\text { Máxima: 36 minutos 33 segundos } \\
\text { Promedio aproximado: 36 minutos 33 segundos }\end{array}$ \\
\hline Estructura de contenidos & Noticias y entrevistas sobre el tema \\
\hline
\end{tabular}

Fig. 12: Podcast Patrimonio Cultural. Fuente: Elaboración propia.

\section{Conclusiones}

El empleo de una herramienta como el podcast para la difusión de contenidos temáticamente referidos al patrimonio cultural es una realidad presente en una considerable cantidad de los países pertenecientes a la esfera castellano parlante. Del total de doce podcasts analizados su distribución por países es: España (cuatro), Colombia (tres), Paraguay, Bolivia, Perú, México y El Salvador (todos ellos con uno).

Se percibe una mezcla de toda clase de podcasters, con la siguiente distribución numérica por tipología: de carácter individual, bien a título estrictamente particular (dos) bien ligados al sector empresarial privado (uno) o al ámbito de la investigación en centros públicos (dos); y de tipo corporativo, con representación de los sectores de los medios de comunicación (tres), educación-formación (dos), público (uno) y 
organizaciones no gubernamentales (uno).

Desde el punto de vista cronológico las primeras experiencias se inician en el año 2014, con los casos de los podcasts Patrimonio Cultural y Artístico de Diputación (España) y Patrimonio Cultural (El Salvador). Al mismo tiempo, a partir de 2019 se advierte un notable incremento en el interés de esta innovación tecnológica para la distribución de esta clase de contenidos, comenzando a partir de ese momento justo la mitad del total de doce podcasts examinados (de ellos, tres corresponden a los primeros 45 días del año 2020).

De los doce podcasts considerados nueve se encuentran incorporados en la plataforma iVoox y únicamente tres en SoundCloud (en ningún caso se produce una duplicación de plataformas). Este desequilibrio es bastante lógico ante la concatenación de dos circunstancias: el superior peso específico que iVoox tiene en el entorno castellano parlante (lengua donde es la mayor plataforma de podcasting a nivel mundial) y la identificación de SoundCloud con una plataforma más centrada en la temática musical que en los contenidos de carácter cultural.

En líneas generales se detecta una muy escasa continuidad (y por consiguiente una inexistente actualización de contenidos) en el empleo de esta herramienta por parte de los diferentes generadores de contenidos. En cuatro de los casos nos encontramos ante un único podcast que posteriormente no tuvo continuidad y en tres esta cifra se queda en los dos programas. Por otro lado, ONG Coprous (Colombia) es el único que ha superado la barrera de los diez generando un total de doce podcasts.

En definitiva, contar con una lengua común hablada por una comunidad castellano parlante compuesta por 580 millones de personas supone una formidable oportunidad para la puesta en marcha de toda clase de podcasts desde el punto de vista temático. Debido a ello, el recorrido potencial que tiene esta tecnología para ser empleada en la difusión del patrimonio cultural resulta más que notable, por lo que muy posiblemente en el corto plazo se asista a una eclosión de esta clase de contenidos similar a la que se ha producido en otros sectores.

\section{Bibliografía}

ANDERSEN, Paul. What is Web 2.0? Ideas, technologies and implications for education. Bristol: JISC, 2007.

BARBEITO VELOSO, María Luz y PERONA PÁEZ, Juan José. Comunicación organizacional en clave sonora: el caso de Blink, los podcasts de BBVA. El Profesional de la Información [en línea]. 2019, vol. 28, n. 5, pp. 1-9. [Fecha de consulta: 27-01-2020]. Disponible en <http://www.elprofesionaldelainformacion.com/contenidos/2019/sep/barbeitoperona.pdf $>$.

BELLMAN, Barry. Computer Networks as Social Networks. Science [en línea]. 2001, vol. 293, n. 5537, pp. 2031-2034. [Fecha de consulta: 18-01-2020]. Disponible en 
<https://science.sciencemag.org/content/293/5537/2031>.

BERNAL BRAVO, César y ANGULO RASCO, Félix. Interacciones de los jóvenes andaluces en las redes sociales. Comunicar [en línea]. 2012, vol. 40, pp. 25-30. [Fecha de consulta: 17-01-2020]. Disponible en $<$ https://www.revistacomunicar.com/index.php?contenido=detalles\&numero=40\& articulo $=40-2013-04>$.

BERRY, Richard. A Golden Age of Podcasting? Evaluation Serial in the Context of Podcast Histories. Journal of Radio \& Audio Media [en línea]. 2015, vol. 22, n. 2, pp. 170-178. [Fecha de consulta: 25-01-2020]. Disponible en <https://www.tandfonline.com/doi/abs/10.1080/19376529.2015.1083363>.

$\mathrm{BIRCH}$, Heather. Potential of SoundCloud for mobile learning in music education: a pilot study. International Journal of Mobile Learning and Organisation [en línea]. 2017, vol. 11, n. 1, pp. 30-40. [Fecha de consulta: 31-01-2020]. Disponible en <https://www.researchgate.net/profile/Heather_Birch3/publication/312011543_Po tential_of_SoundCloud_for_mobile_learning_in_music_education_A_pilot_study/l

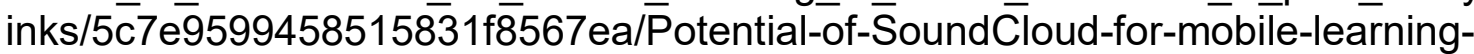
in-music-education-A-pilot-study.pdf $>$.

BIRD, Elizabeth. Are We All Producers Now? Convergence and Media Audience Practices. Cultural Studies [en línea]. 2011, vol. 25, n. 4-5, pp. 502-516. [Fecha de consulta: 13-01-2020]. Disponible en $<$ https://www.tandfonline.com/doi/abs/10.1080/09502386.2011.600532>.

BLANCO HERNÁNDEZ, María; RODERO ANTÓN, Emma y CORREDERA DE COLSA, Luis Enrique. Evolución del conocimiento y consumo de podcasts en España e Iberoamérica. Trípodos [en línea]. 2013, vol. 33, pp. 53-72. [Fecha de consulta: 17-01-2020]. Disponible en <http://www.tripodos.com/index.php/Facultat_Comunicacio_Blanquerna/article/vi ew/97/277>.

BOFIL, Leslie. Constructivism and Collaborating Using Web 2.0 Technology. Journal of Applied Learning Technology. 2013, vol. 3, n. 2, pp. 31-37.

BOYD, Danah y ELLISON, Nicole. Social Network Sites: Definition, History and Scholarship. Journal of Computer-mediated Comunication [en línea]. 2007, vol. 13, n. 1, pp. 210-230. [Fecha de consulta: 19-01-2020]. Disponible en <https://academic.oup.com/jcmc/article/13/1/210/4583062>.

BRIGGS, Mark. Periodismo 2.0. Austin: Knight Foundation, 2007.

CARUSO, Shirley. Towards Understanding the Role of Web 2.0 Technology in SelfDirected Learning And Job Performance. Contemporary Issues in Education Research [en línea]. 2018, vol. 11, n. 3, pp. 89-98. [Fecha de consulta: 23-012020]. Disponible <https://clutejournals.com/index.php/CIER/article/view/10180/10254>. 
CEBRIÁN HERREROS, Mariano. Desarrollo del periodismo en Internet. Sevilla: Comunicación Social, 2010.

CHACÓN, Carmen y PÉREZ, Clevia. El podcast como innovación en la enseñanza del inglés como lengua extranjera. Pixel-Bit Revista de Medios y Educación [en línea]. 2011, vol. 39, pp. 41-54. [Fecha de consulta: 22-01-2020]. Disponible en <https://recyt.fecyt.es/index.php/pixel/article/view/61449/37462>.

COLEMAN, David y LEVINE, Stewart. Collaboration 2.0: technology and best practices for successful collaboration in a Web 2.0 world. Silicon Valley: Happy About, 2008.

CORTIZO, José Carlos. Podcasting, un mercado que está despegando a lo grande. En Digital [en línea]. 2018, 30 de mayo. [Fecha de consulta: 18-01-2020]. Disponible en <https://en.digital/blog/podcasting-un-mercado-que-estadespegando-a-lo-grande/854>.

DI NUCCI, Darcy. Fragmented Future. Print Magazine [en línea]. 1999, vol. 53, n. 4, pp. 32, pp. 221-222. [Fecha de consulta: 23-01-2020]. Disponible en <http://darcyd.com/fragmented_future.pdf>.

Edison Research. The Podcast Consumer 2017 [en línea]. 2017. [Fecha de consulta: 24-01-2020]. Disponible en <http://www.edisonresearch.com/wpcontent/uploads/2017/04/Podcast-Consumer-2017.pdf>.

FUMERO, Antonio y ROCA, Genís. Web 2.0. Madrid: Fundación Orange, 2007.

GARCÍA GALERA, María del Carmen y HOYO HURTADO, Mercedes del. Redes sociales, un medio para la movilización juvenil. Zer Revista de Estudios de Comunicación [en línea]. 2013, vol. 18, n. 34, pp. 111-125. [Fecha de consulta: 18-01-2020]. Disponible

en <https://www.ehu.eus/ojs/index.php/Zer/article/view/10649/9887>.

GARCÍA MARÍN, David y APARICI MARINO, Roberto. Nueva comunicación sonora. Cartografía, gramática y narrativa transmedia del podcasting. El Profesional de la Información [en línea]. 2018, vol. 27, n. 5, pp. 1071-1081. [Fecha de consulta: 26-01-2020]. <http://www.elprofesionaldelainformacion.com/contenidos/2018/sep/11.html>.

GEETS, Kevin. El éxito del 'podcast' y cómo tu marca puede aprovecharlo. MarketingNews [en línea]. 2019, 8 mayo. [Fecha de consulta: 27-01-2020]. Disponible en $<$ https://www.marketingnews.es/tecnologia/opinion/1132179054405/exito-delpodcast-y-marca-puede-aprovecharlo.1.html>.

GONZÁLEZ CONDE, Julia. La ciberradio. Nueva alternativa de futuro para la radio. Revista de Estudios de Juventud [en línea]. 2010, vol. 88, pp. 51-62. [Fecha de consulta: 18-01-2020]. 
<http://www.injuve.es/sites/default/files/RJ88-05.pdf>.

GRAU IBARRA, Katia. SoundCloud: sus posibilidades y limitaciones como una plataforma de distribución de podcast educativos. Revista de Educación a Distancia [en línea]. 2019, vol. 59, pp. 1-16. [Fecha de consulta: 30-01-2020]. Disponible en <https://revistas.um.es/red/article/view/378031/272911>.

GROSSECK, Gabriela. To use or not to use Web 2.0 in higher education? Procedia Social and Behavioural Sciences [en línea]. 2009, vol. 1, n. 1, pp. 478-482. [Fecha de consulta: 27-01-2020]. Disponible en <https://www.sciencedirect.com/science/article/pii/S1877042809000895?via\%3Di hub>.

GUTIÉRREZ PORLÁN, Isabel y RODRÍGUEZ CIFUENTES, María Trinidad. Haciendo historia del podcast. Referencias sobre su origen y evolución. En: SOLANO FERNÁNDEZ, Isabel (ed.). Podcast educativo. Aplicaciones y orientaciones del m-learning para la enseñanza. Murcia: MAD Eduforma, 2010, pp. 17-32.

HAMMERSLEY, Ben. Audible revolution. The Guardian [en línea]. 2004, 12 de febrero. [Fecha de consulta: 05-01-2020]. Disponible en <https://www.theguardian.com/media/2004/feb/12/broadcasting.digitalmedia>.

HARRIS, Albert y REA, Alan. Web 2.0 and Virtual World Technologies: A Growing Impact on IS Education. Journal of Information Systems Education [en línea]. 2019, vol. 20, n. 2, pp. 137-144. [Fecha de consulta: 03-01-2020]. Disponible en $<$ https://aisel.aisnet.org/cgi/viewcontent.cgi?article=1271\&context=jise>.

HAYTHORNTHWAITE, Caroline. Social networks and Internet connectivity effects. Information, Communication \& Society [en línea]. 2005, vol. 8, n. 2, pp. 125-147. [Fecha de consulta: 17-01-2020]. Disponible en <https://www.tandfonline.com/doi/abs/10.1080/13691180500146185>.

Instituto Cervantes. El español: una lengua viva. Informe 2019 [en línea]. Madrid: Instituto Cervantes, 2019. 96 p. [Fecha de consulta: 19-02-2020]. Disponible en <https://www.cervantes.es/imagenes/File/espanol_lengua_viva_2019.pdf>.

ISLAS CARMONA, José Octavio. El prosumidor. El actor comunicativo de la sociedad de la ubicuidad. Palabra Clave [en línea]. 2008, vol. 11, n. 1, pp. 29-39. [Fecha de consulta: 19-01-2020]. Disponible en <https://www.redalyc.org/pdf/649/64911103.pdf>.

KAMEL BOULOS, Maged y WHEELER, Steve. The emerging Web 2.0 social software: an enabling suite os sociable technologies in health and health care education. Health Information and Libraries Journal [en línea]. 2007, vol. 24, pp. 2-23. [Fecha de consulta: 25-01-2020]. Disponible en <https://onlinelibrary.wiley.com/doi/full/10.1111/j.1471-1842.2007.00701.x>. 
KANG, Myunghwa y GRETZEL, Ulrike. Effects of podcast tours on tourist experiences in a national park. Tourism Management [en línea]. 2012, vol. 33, n. 2, pp. 440-455. [Fecha de consulta: 25-01-2020]. Disponible en <https://www.sciencedirect.com/science/article/abs/pii/S0261517711001075?via $\% 3$ Dihub>.

Kantar. Media Trends \& Predictions 2020 [en línea]. 2019. [Fecha de consulta: 30-012020]. Disponible en <https://kantar.turtl.co/story/media-trends-and-predictions2020-es/page/1?teaser=true>.

LARA GONZÁLEZ, Alicia de y CAMPO CAÑIZARES, Elpidio del. El podcast como medio de divulgación científica y su capacidad para conectar con la audiencia. Revista Mediterránea de Comunicación [en línea]. 2018, vol. 9, n. 1, pp. 347359. [Fecha de consulta: 19-01-2020]. Disponible en <https://www.mediterraneacomunicacion.org/article/view/2018-v9-n1-El-podcast-como-medio-dedivulgacion-cientifica-y-su-capacidad-para-conectar-con-la-audiencia> .

LIBEN-NOWELL, David y KLEINBERG, Jon. The link-prediction problem for social networks. Journal of the American Society for Information Science and Technology [en línea]. 2007, vol. 58, n. 7, pp. 1019-1031. [Fecha de consulta: 2201-2020]. Disponible $<$ https://onlinelibrary.wiley.com/doi/abs/10.1002/asi.20591>.

LOCKER, Melissa. .Apple's podcast just topped 50 billion-all time downloads and streams. Fastcompany [en línea]. 2018, 25 de abril. [Fecha de consulta: 17-012020]. Disponible en <https://www.fastcompany.com/40563318/apples-podcastsjust-topped-50-billion-all-time-downloads-and-streams>.

LOFTIAN, Reza y BUSSO, Carlos. Building Naturalistic Emotionally Balanced Speech Corpus by Retrieving Emotional Speech from Existing Podcast Recordings. IEEE Transactions on Affective Computing [en línea]. 2019, vol. 10, n. 4, pp. 471-483. [Fecha de consulta: 29-01-2020]. Disponible en $<$ https://ieeexplore.ieee.org/document/8003425>.

LÓPEZ VILLAFRANCA, Paloma. Estudio de casos de la ficción sonora en la radio pública, RNE, y en la plataforma de podcast del Grupo Prisa en España. Disertaciones [en línea]. 2019, vol. 12, n. 2, pp. 65-78. [Fecha de consulta: 2202-2020]. <https://revistas.urosario.edu.co/index.php/disertaciones/article/view/6547/7299>

MARTOS RUBIO, Ana. Redes Sociales. Madrid: Anaya Multimedia, 2010.

MCHANEY, Roger. The New Digital Shoreline: How 2.0 and Milennials Are Revolutionizing Higher Education. Sterling: Stylus Publishing, 2012.

MORENO CAZALLA, Lourdes. Podium podcast, cuando el podcast tiene acento español. Prisma Social [en línea]. 2017, vol. 18, pp. 334-364. [Fecha de consulta: 
<https://dialnet.unirioja.es/servlet/articulo?codigo=6220235>.

MCNAMARA, Jim. Public communication practices in the Web 2.0-3.0 mediascape: The case of PRevolution. Prism [en línea]. 2013, vol. 7, n. 3, pp. 1-13. [Fecha de consulta: 29-01-2020]. Disponible en <https://www.prismjournal.org/uploads/1/2/5/6/125661607/v7-no3-a3.pdf>.

NEWMAN, Nic y GALLO, Nathan. News Podcasts and the Opportunities for Publishers. Oxford: Reuters Institute, 2019.

O'REILLY, Tim. What is Web 2.0. Design Patterns and Business Models for the Next Generation of Software. O'Reilly [en línea]. 2005. [Fecha de consulta: 24-012020]. Disponible en <http://www.oreillynet.com/pub/a/oreilly/tim/news/2005/09/30/what-is-web20.html>.

ORTIZ SOBRINO, Miguel Ángel. Radio y post-radio en España: una cohabitación necesaria y posible. Área Abierta [en línea]. 2012, vol. 12, n. 2, pp. 1-16. [Fecha de consulta: 10-01-2020]. Disponible en <https://revistas.ucm.es/index.php/ARAB/article/view/39637>.

PARRA VALCARCE, David. Periodismo ciudadano y cambio social: análisis de iniciativas colaborativas en el ámbito del periodismo de investigación. Estudios sobre el Mensaje Periodístico [en línea]. 2017, vol. 23, n. 1, pp. 135-146. [Fecha de consulta: 20-01-2020]. Disponible en $<$ https://revistas.ucm.es/index.php/ESMP/article/view/55587/50445>.

PARRA VALCARCE, David y MARTÍNEZ ARIAS, Santiago. Tecnologías de la Gestión Periodística de la Información Digital. Conceptos básicos. Madrid: Los Autores, 2018.

Podtrac. Podcast industry audience rankings [en línea]. 2020. [Fecha de consulta: 10-02-2020]. Disponible en <http://analytics.podtrac.com/industry-rankings/>.

SELLAS GÜELL, Antoni. La voz de la Web 2.0. Análisis del contexto, retos y oportunidades del podcasting en el marco de la comunicación sonora [en línea]. Barcelona: Universitat Internacional de Catalunya, 2009. [Fecha de consulta: 1902-2020]. Disponible en <https://www.tdx.cat/handle/10803/9351\#page=1>.

SELLAS GÜELL, Antoni. El uso del podcasting como herramienta de la identidad corporativa en la campaña del 90 aniversario de Iberia. El caso del podcast Aerolínea Momentos. Communication Papers [en línea]. 2018, vol. 7, n. 15, pp. 155-170. [Fecha de consulta: 22-01-2020]. Disponible en <https://revistes.udg.edu/communication-papers/article/view/22189>.

SHANTIKUMAR, Saran. From lecture theatre to portable media: students' perceptions of an enhanced podcast for revision. Medical Teacher [en línea]. 2009, vol. 31, n. 6, pp. 535-538. [Fecha de consulta: 29-01-2020]. Disponible en 
<https://www.tandfonline.com/doi/abs/10.1080/01421590802365584>.

STERNE, Jonathan. The mp3 as cultural artifact. New Media \& Society [en línea]. 2006, vol. 8, n. 5, pp. 825-842. [Fecha de consulta: 11-01-2020]. Disponible en: <https://journals.sagepub.com/doi/10.1177/1461444806067737>.

SULLIVAN, John L. The Platforms of Podcasting: Past and Present. Social Media \& Society [en línea]. 2019, vol. 5, n. 4, pp. 1-12. [Fecha de consulta: 28-01-2020]. Disponible <https://journals.sagepub.com/doi/pdf/10.1177/2056305119880002>.

TAPSCOTT, Don. The Digital Economy. Promise and Peril in The Age of Networked Intelligence. Nueva York: McGraw-Hill, 1995.

TOFFLER, Alvin. The Third Wave. Nueva York: Bantam Books, 1980.

VILLANUEVA, Xavi. Qué es iVoox con Juan Ignacio Solera. Abismo FM [en línea]. 2019. [Fecha de consulta: 30-01-2020]. Disponible en $<$ https://abismofm.com/que-es-ivoox/>.

VIÑAS, Meritxell. SoundCloud: El audio como como potente herramienta en educación. Totemguard [en línea]. 2012. [Fecha de consulta: 28-01-2020]. Disponible en <https://www.totemguard.com/aulatotem/2012/06/soundcloud-elaudio-como-potente-herramienta-en-educacion/>. 